\title{
Epithelial Barrier Leak in Gastrointestinal Disease and Multiorgan Failure
}

\author{
Christopher P. Farrell ${ }^{1, *}$, Maura Barr ${ }^{2}$, James M. Mullin ${ }^{1}$, Leah Lande ${ }^{3}$ and Marc Zitin ${ }^{1}$ \\ ${ }^{I}$ Department of Gastrointestinal Medicine, Lankenau Hospital, Wynnewood, PA 19096, USA \\ ${ }^{2}$ Department of Internal Medicine, Lankenau Hospital, Wynnewood, PA 19096, USA \\ ${ }^{3}$ Department of Pulmonary and Critical Care Medicine, Lankenau Hospital, Wynnewood, PA 19096, USA
}

\begin{abstract}
There are a myriad of clinical conditions whose disease etiology involves leakiness of an epithelial and/or endothelial barrier. To underscore the medical usefulness of agents that can augment barrier function, the theme of this special issue, two disease areas are highlighted. The first is the phenomenon of "leaky gut" which can play relatively obvious roles in gastrointestinal diseases such as celiac disease, Crohn's disease, and ulcerative colitis. It may play less obvious roles in seemingly unrelated illnesses such as autism, diabetes and certain microbial infectious diseases. A second disease area where barrier compromise plays a major role is multi-organ failure, a major cause of death in ICUs. Multi-organ failure highlights the important effect that the immune system and oxidative stress can have on barrier elements. The use of barrier enhancing and protecting agents in nutritional feeding in ICUs should be an area of intense interest in the future.
\end{abstract}

Keyword: Tight junctions, inflammatory bowel disease, multiorgan dysfunction syndrome.

\section{BARRIER LEAK IN THE GI TRACT}

The gastrointestinal (GI) tract is a complex system that separates an individual from their environment through an intestinal barrier. There is a complex association of digestive enzymes and barrier elements ranging from mucus to epithelial tight junctions that formulate this separation from the outside world [1]. When this barrier becomes compromised, increased intestinal permeability occurs, allowing for the penetration of toxins and other pathogenic material. This process of a "leaky gut" can yield tissue injury and the spread of systemic disease [2]. A number of GI disorders perpetuate or derive from intestinal barrier disruption, leading to increased permeability. Other systemic conditions can act indirectly on the GI tract, also altering permeability and barrier function.

Celiac disease is an autoimmune chronic enteropathy caused in part by eating gluten products such as wheat, barley, and rye. Ingestion of such material causes a flattening of duodenal villi, normally used to absorb nutrients. A wide variety of symptoms can occur, ranging from asymptomatic anemia to chronic diarrhea and failure to thrive. The diagnosis can be made through serologic testing, in the form of a tissue transglutaminase (TTG) or anti-endomysial antibody or from pathological examination of duodenal biopsies from endoscopy, illustrating flattened villi and lymphocyte infiltration. In this condition, gliadin-containing products also disrupt intestinal barrier function. Transepithelial resistance is decreased and paracellular permeability is increased

*Address correspondence to this author at the Lankenau Hospital GI Fellows Office, $1^{\text {st }}$ Floor 100 E. Lancaster Ave. Wynnewood, PA 19096, USA; Tel: 215-850-2575; Fax: 484-476-2077; E-mail: cfarrell22@hotmail.com as a result of altered expressions of tight junction proteins, including occludin, claudins, and ZO-1 [3, 4]. The treatment for celiac disease is a gluten-free diet. While this sounds simple, it is a life-changing remedy that can provide difficult adherence. Compliance with a gluten-free diet reverses the effects of celiac disease, and can resolve the barrier leak and intestinal permeability.

Inflammatory bowel disease (IBD) consists of Crohn's disease (CD) and ulcerative colitis (UC). IBD is thought to be due to an interaction between the environment and an inappropriate host immune response to genetically predisposed individuals [5]. Both diseases are autoimmune inflammatory disorders that affect the GI mucosa, but are independent from one another. Nonetheless, both forms of IBD demonstrate a partial loss of tight junction barrier function, producing transepithelial leak and increased passage of material from the gut lumen [6]. Sugar absorption tests have proven this phenomenon, more markedly when possessing active disease; however, still elicited in disease remission [7].

CD can affect any portion of the GI tract from the mouth to the anus, most often affecting the terminal ileum. The inflammation in CD can extend through all layers of the bowel wall, causing transmural injury. Its ability to penetrate into the submucosa allows for the potential formation of fistulous tracts and abscesses. Strictures can also form from prolonged inflammation and scarring, and present as an intestinal obstruction. CD has been shown to display a reduction in tight junction strands, strand breaks, and altered tight junction protein composition, leading to increased intestinal permeability [8]. Tumor necrosis factor (TNF) alpha is upregulated in patients with CD. TNF-alpha has demonstrated effects on tight junction permeability through NF-kappa B activation 
[9]. Treatment of CD with anti-TNF-alpha agents has been shown to dramatically decrease intestinal inflammation and permeability, helping restore gut barrier function [10].

UC invariably involves the rectum and extends proximally throughout the colon, and inflammation is generally limited to the mucosa. UC is more of a diffuse colitis, affecting colonic barrier and permeability in both chronic and acute colitis [11]. Transepithelial leaks can occur early in UC as a result of apoptotic foci from microerosions or tight junction protein alterations with an increase in claudin- $2[8,12]$. Controlling the interleukin-induced inflammation in UC also allows for a regaining of intestinal barrier function and a decrease in permeability. Barrier dysfunction in IBD is obvious, but it remains unclear whether or not it is a primary contributor to disease or just a consequence of mucosal inflammation [13].

Another form of microscopic colitis exists that presents mainly as chronic diarrhea, known as collagenous or lymphocytic colitis. The diagnosis is made by microscopic examination of random colonic biopsies via colonoscopy in a symptomatic patient. A disturbance in colonic mucosal permeability is even seen in this mild colitis, which affects mucosa only on a microscopic level [14].

Helicobacter pylori (H. pylori) is a spiral, gram-negative bacteria that affects the upper GI tract. $\mathrm{H}$. pylori resides in the human stomach and is an acquired infection. If left untreated, the bacteria can progress to form a chronic gastritis, gastric or duodenal ulcers, and even gastric cancer. Infected stomachs display an increase in mucosal permeability [15]. Even asymptomatic patients elicit a disruption in gastric mucosal integrity, altering its barrier function [16]. H. pylori has been shown to disturb tight junctions by phosphorylating myosin light chains and regulating claudin- 4 and claudin-5 [17]. Other studies have linked ammonium production by $\mathrm{H}$. pylori causing a modification in tight junctions [18]. All patients with $\mathrm{H}$. pylori should be treated with a proton pump inhibitor and antibiotic therapy in an attempt to eradicate the bacteria, both resolving this leak and preventing future potential complications.

Other disease processes that span the realm of gastroenterology can also elicit a GI leak, even though they do not directly act on the GI mucosa. One such phenomenon is that of acute pancreatitis. The two most common causes for acute pancreatitis are gallstones and alcohol intake. Patients present with severe epigastric pain and can be extremely ill with multiorgan failure and even progress to death. Acute pancreatitis has shown a decrease in gut absorptive capacity with an increase in gut permeability $[19,20]$. The intestinal mucosa has shown partial breakdown via accelerated epithelial apoptosis, allowing for increased permeability and bacterial translocation [21]. The degree of barrier dysfunction correlates with the severity and duration of pancreatitis [22]. Therefore, if a therapeutic intervention to help resolve the inflammation can be performed, such as an endoscopic retrograde cholangiopancreatography (ERCP) to extract impacted gallstones, both the extent of disease and permeability disorder can be decreased. Aggressive treatment and management of acute pancreatitis is critical to minimize comorbidities and increase survival.
Intestinal permeability increase is also present in patients with advanced liver disease, mainly cirrhosis [23, 24]. The highest leak is observed in alcoholic cirrhotics compared to those with cirrhosis from viral hepatitis, while patients with just chronic viral hepatitis without cirrhosis did not exhibit an increased GI leak compared to controls [25]. This alteration in barrier function has been hypothesized to be due to toxic effects of alcohol and portal hypertensive gastropathy [26]. The effects of alcohol and its metabolites on epithelial barriers has been intensively investigated [27]. These permeability derangements are more pronounced in patients with more advanced liver decompensation, who possess ascites and/or a prior history of spontaneous bacterial peritonitis (SBP) [28]. SBP occurs from bacterial translocation into ascitic fluid from an overall breakdown in GI and vascular barrier function. Patients who experience even just one episode of SBP require antibiotic prophylaxis either lifelong or until they receive a liver transplant.

Primary biliary cirrhosis (PBC) is an autoimmune disease that affects the biliary system and can progress to cirrhosis. This condition is more prevalent in females and can present with just fatigue and pruritis. GI permeability increase has been seen in PBC, and is thought to be due to an epithelial dysfunction, rather than portal hypertension, as seen in cirrhotics without PBC [29]. These findings suggest a more systemic, and possibly autoimmune-based, etiology of GI leak compared to others with liver disease. PBC has a slow, indolent course in most cases, and is only curable through a liver transplantation. Otherwise, the treatment mainly consists of ursodeoxycholic acid to delay the progression of liver disease.

Hepatitis C (HCV) is a viral infection that is acquired parenterally, most commonly through blood transfusions and intravenous drug use. The main organ targeted with $\mathrm{HCV}$ is the liver, and chronic infection can lead to cirrhosis. As discussed previously, cirrhosis causes an increase in intestinal permeability, while chronic hepatitis does not. However, $\mathrm{HCV}$ does interact with tight junction proteins in a different manner. Claudin-1 is considered a co-receptor for $\mathrm{HCV}$, and is essential for the entry of HCV into hepatocytes [30, 31]. Occludin has also been implicated as playing a critical role in $\mathrm{HCV}$ entry into the liver, through an intracellular interaction with E2 glycoprotein [32]. These findings help provide new insights into the mechanisms of action of HCV and potential future treatment therapies.

Systemic inflammation induces intestinal permeability increase, as seen in acute pancreatitis, but is also found in patients suffering from sepsis and septic shock. Increased GI leak in sepsis and multiorgan failure is due to an epithelial dysfunction and possibly caused by an inflammationinduced, cytokine-mediated, paracellular permeability increase [33, 34]. One small study found an increase in colorectal permeability that correlated with the degree of sepsis and shock [35].

Different infectious etiologies have been linked to increased GI leak; however, one unique bacterium is Listeria monocytogenes. It is indicative of the preferential targeting of the tight junctions and cell adhesion sites by many pathogens [36]. This is a food-borne, gram-positive bacterium that causes human listeriosis, and must cross the intestinal epithe- 
lial barrier to attack other organ systems in the human body [37]. L. monocytogenes permeates the intestinal barrier in an unusual way, through the interaction of its surface protein, internalin, with the enterocyte receptor E-cadherin [38]. Altered localization of the tight junction protein, $\mathrm{ZO}-1$, is involved in the subsequent tight junction disruption [39]. This allows the passage of the bacteria from the GI tract through the intestine, and can lead to a possible targeting of the placental or blood-brain barrier.

Human immunodeficiency virus (HIV) and acquired immunodeficiency syndrome (AIDS) have shown alterations in small intestinal transit, absorption, and permeability. The function of the absorptive surface of the intestine decreases as HIV progresses [40, 41]. An impairment in intestinal permeability occurs as HIV develops into AIDS, which can elicit a significant enteric protein loss [42, 43].

While autoimmune diseases that primarily affect the GI lumen alter epithelial barrier function, other autoimmune disorders can yield similar findings. Diabetes mellitus type 1 is associated with mucosal ultra-structural alterations, causing an increase in intestinal permeability [44, 45]. However, this phenomenon is not seen in diabetes mellitus type 2 , an acquired condition that is not of autoimmune etiology [46]. Allergic reactions to food also alter intestinal permeability, likely on both a mucosal and systemic basis [47].

\section{BARRIER LEAK IN MULTIORGAN FAILURE}

Multiorgan failure, or multiorgan dysfunction syndrome (MODS), is the most common cause of death in critically ill patients and has been described as "death in slow motion" $[48,49]$. MODS is the final common pathway that can follow the systemic inflammatory response syndrome (SIRS) in situations such as sepsis, hemorrhagic shock, burn injury, pancreatitis, trauma and even cardiac surgery. The most commonly affected organs are the lungs, GI tract, kidneys, and liver; the major epithelial barriers in the body. The pathogenesis behind the deterioration and eventual failure of organ systems that occurs during this process remains unknown, but is believed to be the end result of an inflammatory response on overdrive. This overactive immune response leads to widespread cellular barrier dysfunction [49]. There have been many theories proposed as to what initiates and propagates this destructive process. When these physiologic states introduce a flood of inflammatory mediators into the bloodstream they can cause a sustained activation of vascular endothelium, initiating a dysregulated cascade that can ultimately lead to irreversible tissue damage and death [50]. TNF alpha and other cytokine-mediated epithelial apoptosis and tight junction dysregulation are well-documented phenomena [51, 52].

In recent times, researchers have hypothesized that the gut, more specifically an increase in GI permeability, acts as the "motor" behind MODS in critical illness [53]. Various theories have been discussed regarding the mechanism of this increase in gut barrier function permeability, initially concluding that hypoxia/reperfusion injury in the gut leads directly to diminished barrier function, bacterial translocation across the gut, and the subsequent entrance of toxic substances into circulation. Successive studies have proven that the mechanism of barrier dysfunction, and the ensuant body- wide organ dysfunction that can occur, may be a more complex interplay of ischemia/reperfusion, altered tight junctions, cytokine production, epithelial cell apoptosis, and bacterial translocation.

The exact mechanism behind organ dysfunction in MODS remains elusive. A common theme in the conditions that can lead to MODS is that the initial insult leads to splanchnic hypoperfusion. This hypoperfusion is often subclinical, caused by vasoconstriction of the splanchnic vessels in response to physical stress and hypovolemia, even prior to changes in a patient's heart rate or blood pressure [54]. Whether it is due to the ischemia itself or reperfusion injury that follows, the gut then becomes a major site for proinflammatory cytokine production [55]. These inflammatory mediators and other "gut-derived tissue injurious factors", as well as bacterial endotoxins, are then present to cause a subsequent loss of gut barrier function. This cascade of events may then be followed by a cycle of bacterial translocation across the epithelial barrier and further activation of the innate immune response. The production and release of these cytokines and toxins may have direct or indirect systemic effects that we classify under the umbrella term "MODS" $[49,55]$.

Just how inflammatory mediators and bacterial endotoxins lead to an alteration of gut barrier function is still being debated. Barrier function in the body is regulated in part by tight junctions, comprised of a complex of proteins between the apical components of epithelial cells that limit passive diffusion across the paracellular pathway and prevent contamination of the body's internal environment with toxic substances that enter from the external world [56-58]. There is increasing evidence that pro-inflammatory stimuli such as TNF-alpha and IL-1 beta can induce changes in occludin and ZO-1 expression and localization within the cell. Nitric oxide (NO) has been implicated in the reorganization and decreased production of the tight junction proteins via up-regulated inducible NO synthase (iNOS) expression and increased NO production in response to cytokines or bacteria. Cultured epithelial cells exposed to sera from MODS patients demonstrated increased permeability as well as decreased expression levels and disordered distribution of tight junction proteins within the cell. This corresponded to elevated NO and increased iNOS expression in epithelial monolayers treated with the MODS serum [59].

There is evidence from studies performed by Fink and Delude that the presence of lipopolysaccharide (LPS) and endotoxemia may increase intestinal permeability under similar iNOS dependent pathways [49]. Systemic inflammation, a milieu introduced by LPS injection simulating sepsis, is associated with hepatobiliary epithelial barrier dysfunction in mice. A similar reaction is witnessed in the bronchoalveolar epithelial barrier with the induction of endotoxemia leading to increased lung permeability, mediated at least in part by iNOS-dependent NO synthesis [49]. This process is associated with decreased occludin and ZO-1 expression and staining in both hepatic and lung tissue extracts. This provides a possible mechanism for which a systemic inflammatory reaction, reproduced in these studies by induction of endotoxemia by LPS injection, can lead to distant organ injury like cholestatic liver dysfunction and the pulmonary 
edema associated with acute lung injury (ALI) and acute respiratory distress syndrome (ARDS). This barrier breakdown and subsequent tissue damage may be extrapolated to other organs, such as the kidneys, that are also often damaged in MODS.

Once an alteration in the tight junction has been established, bacterial translocation and the invasion by microbes of previously sterile tissue compartments can lead to an activation of the innate immune response [49]. Translocation describes the entrance of enteric bacteria as well as LPS endotoxins and other antigenic molecules through the gut barrier and into the rest of the body via the bloodstream or lymphatics [55]. This can lead to further barrier dysfunction, tissue edema, and organ dysfunction at distant sites by mechanisms that have not been fully described. The propagation of the cycle of inflammation by bacterial translocation and the movement of other gut-derived cytokines and toxins through the gut is known as the "leaky gut hypothesis" [53]. In the past, theories describing gut barrier dysfunction as essential to the development of MODS by these mechanisms have been confounded by a lack of evidence demonstrating bacterial endotoxins in the portal circulation of these patients. The failure of anti-endotoxin therapy and selective digestive tract antibiotic decontamination to improve survival in MODS was also perplexing [60,61]. This begs the question, how do patients with MODS acquire gram negative bacteremia without a focal source of infection? The answer may be that the endotoxins and other injurious inflammatory factors exit the gut via the mesenteric lymphatics. This was evidenced by studies showing that rats experiencing trauma and hemorrhagic shock developed ALI in the form of an increase in pulmonary vascular endothelial permeability, and this response was eliminated by ligation of the lymphatic ducts. Interestingly, the ALI was associated with an increased iNOS-derived nitric oxide level in rats, correlating with prior studies demonstrating a role of iNOS in barrier dysfunction and leaky tight junctions [61]. Other studies indicated similar findings with burn-induced lung and cardiac injury $[62,63]$.

The understanding that gut barrier dysfunction plays a role in perpetuating barrier dysfunction on a grand scale in the form of MODS allows for a focus on possible therapeutic interventions. Maintaining the integrity of endothelial and epithelial barriers may lead to decreased morbidity and mortality. Targeting the inflammatory cascade with anti-cytokine antibodies and systemic glucocorticoids has in the past failed to prevent organ injury [64]. An interesting and novel approach that was proven in the PROWESS trial to improve mortality in severe sepsis with multi-organ dysfunction is the use of activated Protein C (APC) [65]. It is not clear through which mechanisms APC improves mortality, and its actions on the endothelium and epithelium are complex. There is evidence from two in-vitro studies of direct modulation of the endothelial monolayer leading to increased cell-cell contact and decreased barrier permeability via cytoskeletal rearrangement and strengthening of endothelial tight junctions [64].

An improvement in barrier function with the use of probiotic therapy has also been proposed as a potential therapeutic target in MODS. One study of critically ill patients receiving enteral feedings showed a reduction in intestinal permeability in patients getting probiotic supplementation over placebo alone. Assuming that the pathogenesis of MODS in some way stems from an alteration in gut barrier function and increased permeability, the use of probiotics may then be seen as a possible tool in combating this syndrome [66]. Many questions remain, however, regarding the clinical risk/benefit ratio that can be derived from this intervention in light of evidence that critically ill patients in particular are at risk of disseminated fungemia following use of probiotics like Saccharomyces boulardii (S. cerevisiae) [67]. Isolating the critical active components of probiotics, as well as implementation of other barrier enhancing agents such as zinc, polyphenols, rapamycin, and indole, may lead to new therapeutic approaches to MODS in the future.

Barrier dysfunction and transepithelial intestinal permeability has proven to play a pivotal role in the pathophysiology of GI disease and systemic inflammatory disorders. Studies have linked different alterations in tight junctions and immune responses to this process, resulting in advancements in the treatment of an array of diseases. Further research is warranted and ongoing to broaden the understanding of this phenomenon and help aid in future therapies.

\section{CONFLICT OF INTEREST}

None declared.

\section{ACKNOWLEDGEMENTS}

None declared.

\section{REFERENCES}

[1] DeMeo MT, Mutlu EA, Keshavarzian A, Tobin MC. Intestinal permeation and gastrointestinal disease. J Clin Gastroenterol 2002; 34: 385-96.

[2] Farhadi A, Banan A, Fields J, Keshavarzian A. Intestinal barrier: an interface between health and disease. J Gastroenterol Hepatol 2003; 18: 479-97.

[3] Sander GR, Cummins AG, Henshall T, Powell BC. Rapid disruption of intestinal barrier function by gliadin involves altered expression of apical junctional proteins. FEBS Lett 2005; 579: 4851-5.

[4] Clemente MG, De Virgiliis S, Kang JS, et al. Early effects of gliadin on enterocyte intracellular signaling involved in intestinal barrier function. Gut 2003; 52: 218-23.

[5] Salim SY, Söderholm JD. Importance of disrupted intestinal barrier in inflammatory bowel diseases. Inflamm Bowel Dis 2010; [Epub ahead of print].

[6] Edelblum KL, Turner JR. The tight junction in inflammatory disease: communication breakdown. Curr Opin Pharmacol 2009; 9: 715-20.

[7] Welcker K, Martin A, Kölle P, Siebeck M, Gross M. Increased intestinal permeability in patients with inflammatory bowel disease. Eur J Med Res 2004; 9: 456-60.

[8] Mankertz J, Schulzke JD. Altered permeability in inflammatory bowel disease: pathophysiology and clinical implications. Curr Opin Gastroenterol 2007; 23: 379-83.

[9] Ma TY, Iwamoto GK, Hoa NT, et al. TNF-alpha-induced increase in intestinal epithelial tight junction permeability requires NFkappa B activation. Am J Physiol Gastrointest Liver Physiol 2004; 286: G367-76.

[10] Suenaert P, Bulteel V, Lemmens L, et al. Anti-tumor necrosis factor treatment restores the gut barrier in Crohn's disease. Am J Gastroenterol 2002; 97: 2000-4.

[11] Nejdfors P, Wang Q, Ekelund M, et al. Increased colonic permeability in patients with ulcerative colitis: an in vitro study. Scand J Gastroenterol 1998; 33: 749-53. 
[12] Gitter AH, Wullstein F, Fromm M, Schulzke JD. Epithelial barrier defects in ulcerative colitis: characterization and quantification by electrophysiological imaging. Gastroenterology 2001; 121: 1320-8.

[13] McGuckin MA, Eri R, Simms LA, Florin TH, Radford-Smith G. Intestinal barrier dysfunction in inflammatory bowel disease. Inflamm Bowel Dis 2009; 15: 100-13.

[14] Taha Y, Carlson M, Thorn M, Loof L, Raab Y. Evidence of local eosinophil activation and altered mucosal permeability in collagenous colitis. Dig Dis Sci 2001; 46: 888-97.

[15] FukudaY, Bamba H, Okui M, et al. Helicobacter pylori infection increases mucosal permeability of the stomach and intestine. Digestion 2001; 63: 93-6.

[16] Borch K, Sjöstedt C, Hannestad U, Söderholm JD, Franzén L, Mårdh S. Asymptomatic Helicobacter pylori gastritis is associated with increased sucrose permeability. Dig Dis Sci 1998; 43: 749-53.

[17] Fedwick JP, Lapointe TK, Meddings JB, Sherman PM, Buret AG. Helicobacter pylori activates myosin chain light-chain kinase to disrupt claudin-4 and claudin-5 and increase epithelial permeability. Infect Immun 2005; 73: 7844-52.

[18] Lytton SD, Fischer W, Nagel W, Haas R, Beck FX. Production of ammonium by Helicobacter pylori mediates occluding processing and disruption of tight junctions in Caco-2 cells. Microbiology 2005; 151: 3267-76.

[19] Juvonen PO, Alhava EM, Takala JA. Gut permeability in patients with acute pancreatitis. Scand J Gastroenterol 2000; 35: 1314-8.

[20] Liu H, Li W, Li J, Yu W. Early gut mucosal dysfunction in patients with acute pancreatitis. Pancreas 2008; 36: 192-6.

[21] Yasuda T, Takeyama Y, Ueda T, et al. Breakdown of intestinal mucosa via accelerated apoptosis increases intestinal permeability in experimental severe acute pancreatitis. J Surg Res 2006; 135: 18-26.

[22] Nagpal K, Minocha VR, Agrawal V, Kapur S. Evaluation of intestinal mucosal permeability in patients with acute pancreatitis. Am J Surg 2006; 192: 24-8.

[23] Ersöz G, Aydin A, Erdem S, Yüksel D, Akarca U, Kumanlioglu K. Intestinal permeability in liver cirrhosis. Eur J Gastroenterol Hepatol 1999; 11: 409-12.

[24] Cariello R, Federico A, Sapone A, et al. Intestinal permeability in patients with chronic liver diseases: Its relationship with the aetiology and the entity of liver damage. Dig Liver Dis 2010; 42: 200-4.

[25] Kim JW, Jeon WK, Yun JW, et al. Intestinal permeability in patients with viral and alcoholic liver disease. Korean J Gastroenterol 2004; 43: 104-11.

[26] Ancel D, Barraud H, Peyrin-Biroulet L, Bronowicki JP. Intestinal permeability and cirrhosis. Gastroenterol Clin Biol 2006; 30: 460-8.

[27] Basuroy S, Sheth P, Mansbach CM, Rao RK. Acetaldehyde disrupts tight junctions and adherens junctions in human colonic mucosa: protection by EGF and L-glutamine. Am J Physiol Gastrointest Liver Physiol 2005; 289: G367-75.

[28] Scarpellini E, Valenza V, Gabrielli M, et al. Intestinal permeability in cirrhotic patients with and without spontaneous bacterial peritonitis: is the ring closed? Am J Gastroenterol 2010; 105: 323-7.

[29] Di Leo V, Venturi C, Baragiotta A, Martines D, Floreani A. Gastroduodenal and intestinal permeability in primary biliary cirrhosis. Eur J Gastroenterol Hepatol 2003; 15: 967-73.

[30] Evans MJ, von Hahn T, Tscherne DM, et al. Claudin-1 is a hepatitis $\mathrm{C}$ virus co-receptor required for a late step in entry. Nature 2007; 446: 801-5.

[31] Bekker V, O'Brien TR, Chanock S. Population genetics and comparative genetics of CLDN1, a gene involved in hepatitis $\mathrm{C}$ virus entry. Hum Hered 2009; 67: 206-16.

[32] Benedicto I, Molina-Jiménez F, Bartosch B, et al. The tight junction-associated protein occludin is required for a postbinding step in hepatitis C virus entry and infection. J Virol 2009; 83: 8012-20.

[33] Fink MP. Intestinal epithelial hyperpermeability: update on the pathogenesis of gut mucosal barrier dysfunction in critical illness. Curr Opin Crit Care 2003; 9: 143-51.

[34] Heitbrink F, Besselink MG, Renooij W, et al. Systemic inflammation increases intestinal permeability during experimental human endotoxemia. Shock 2009; 32: 374-8.
[35] Jørgensen VL, Nielsen SL, Espersen K, Perner A. Increased colorectal permeability in patients with severe sepsis and septic shock. Intensive Care Med 2006; 32: 1790-6.

[36] Guttman JA, Finlay BB. Tight junctions as targets of infectious agents. Biochim Biophys Acta 2009; 1788: 832-41.

[37] Daniels JJ, Autenrieth IB, Goebel W. Interaction of Listeria monocytogenes with the intestinal epithelium. FEMS Microbiol Lett 2000; 190: 323-8.

[38] Lecuit M. Understanding how Listeria monocytogenes targets and crosses host barriers. Clin Microbiol Infect 2005; 11: 430-6.

[39] Hanajima-Ozawa M, Matsuzawa T, Fukui A. Enteropathogenic Escherichia coli, Shigella flexneri, and Listeria monocytogenes recruit a junctional protein, zonula occludens-1, to actin tails and pedestals. Infect Immun 2007; 75: 565-73.

[40] Tepper RE, Simon D, Brandt LJ, Nutovits R, Lee MJ. Intestinal permeability in patients infected with the human immunodeficiency virus. Am J Gastroenterol 1994; 89: 878-82.

[41] Pernet P, Vittecoq D, Kodjo A, et al. Intestinal absorption and permeability in human immunodeficiency virus-infected patients. Scand J Gastroenterol 1999; 34: 29-34.

[42] Sharpstone D, Neild P, Crane R, et al. Small intestinal transit, absorption, and permeability in patients with AIDS with and without diarrhoea. Gut 1999; 45: 70-6.

[43] Becker K, Lindner C, Frieling T, Niederau C, Reinauer H, Häussinger D. Intestinal protein leakage in the acquired immunodeficiency syndrome. J Clin Gastroenterol 1997; 25: 426-8.

[44] Carratù R, Secondulfo M, de Magistris L, et al. Altered intestinal permeability to mannitol in diabetes mellitus type I. J Pediatr Gastroenterol Nutr 1999; 28: 264-9.

[45] Secondulfo M, Iafusco D, Carratù R, et al. Ultrastructural mucosal alterations and increased intestinal permeability in non-celiac, type I diabetic patients. Dig Liver Dis 2004; 36: 35-45.

[46] Secondulfo M, de Magistris L, Sapone A, Di Monda G, Esposito P, Carratù R. Intestinal permeability and diabetes mellitus type 2. Minerva Gastroenterol Dietol 1999; 45: 187-92.

[47] Ventura MT, Polimeno L, Amoruso AC, et al. Intestinal permeability in patients with adverse reactions to food. Dig Liver Dis 2006; 38: 732-6.

[48] Doig CJ, Sutherland LR, Sandham JD, Fick GH, Verhoef M, and Meddings JB. Increased epithelial permeability is associated with the development of multiple organ dysfunction syndrome in critically ill ICU patients. Am J Respir Crit Care Med 1998; 158: 444-51.

[49] Fink MP, Delude RL. Epithelial barrier dysfunction: A unifying theme to explain the pathogenesis of multiple organ dysfunction at the cellular level. Crit Care Clin North Am 2005; 21: 178-96.

[50] Gaugler MH. A unifying system: does the vascular endothelium have a role to play in multi-organ failure following radiation exposure? BJR Suppl. 2005; 27: 100-5.

[51] Soler AP, Marano CW, Bryans M, Miller RD, Garulacan LA, Mauldin SK, Stamato TD, Mullin JM. Activation of NF-kappa B is necessary for the restoration of the barrier function of an epithelium undergoing TNF-alpha-induced apoptosis. Eur J Cell Biol 1999; 78: 56-66.

[52] Peralta Soler A, Mullin JM, Knudsen KA, Marano CW. Tissue remodeling during tumor necrosis factor-induced apoptosis in LLC-PK1 renal epithelial cells. Am J Physiol 1996; 270: F869-79.

[53] Clark JA, Coopersmith CM. Intestinal crosstalk- a new paradigm for understanding the gut as the "motor" of critical illness. Shock 2007; 28: 384-93.

[54] Krack A, Sharma R, Figulla HR, Anker SD. The importance of the gastrointestinal system in the pathogenesis of heart failure. Europ Heart J 2005; 26: 2368-74.

[55] Gatt M, Reddy BS, Macfie J. Review article: bacterial translocation in the critically ill- evidence and methods of prevention. Aliment Pharmacol Ther 2007; 25: 741-57.

[56] Furuse M. Molecular basis of the core structure of tight junctions. Cold Spring Harb Perspect Biol. 2010 Jan;2(1) :a002907. Review. PubMed PMID: 20182608; PubMed Central PMCID: PMC2827901.

[57] Marchiando AM, Graham WV, Turner JR. Epithelial barriers in homeostasis and disease. Annu Rev Pathol. 2010;5:119-44. Review. PubMed PMID: 20078218. 
[58] Anderson JM, Van Itallie CM. Physiology and function of the tight junction. Cold Spring Harb Perspect Biol. 2009; 1: a002584. Review. PubMed PMID: 20066090; PubMed Central PMCID: PMC2742087.

[59] Zhang JB, Du XG, Zhang H, et al. Breakdown of the gut barrier in patients with multiple organ dysfunction syndrome is attenuated by continuous blood purification: effects on tight junction structural proteins. Int J Artif Organs 2010; 33: 5-14.

[60] Moore FA, Moore EE, Poggetti R, et al. Gut bacterial translocation $v i a$ the portal vein: a clinical perspective with major torso trauma. $\mathrm{J}$ Trauma 1991; 31: 629-39.

[61] Deitch EA, Xu D, Kaiser VL. Role of the gut in the development of injury- and shock-induced SIRS and MODS: the gut-lymph hypothesis, a review. Frontiers Biosci 2006; 11: 520-8.

[62] Magnotti LJ, Xu DZ, Lu Q, Deitch EA. Gut-derived mesenteric lymph: A link between burn and lung injury. Arch Surg 1999; 134: 1333-41.
[63] Sambol JT, White J, Horton JW, Deitch EA. Burn-induced impairment of cardiac contractile function is due to gut-derived factors transported in mesenteric lymph. Shock 2002; 18: 272-6.

[64] Looney MR, Matthay MA. Bench-to-bedside review: the role of activated protein $\mathrm{C}$ in maintaining endothelial tight junction function and its relationship to organ injury. Crit Care 2006; 10: 239-45.

[65] Bernard GR, Vincent JL, Laterre PF, et al. Efficacy and safety of recombinant human activated protein $\mathrm{C}$ for severe sepsis. $\mathrm{N}$ Engl $\mathrm{J}$ Med 2001; 344: 699-709.

[66] Alberda C, Gramlich L, Meddings J, et al. Effects of probiotic therapy in critically ill patients: a randomized, double-blind, placebo-controlled trial. Am J Clin Nutr 2007; 85: 816-23.

[67] Munoz P, Bouza E, Cuenca-Estrella M, et al. Saccharomyces cerevisiae fungemia: an emerging infectious disease. Clin Infect Dis 2005; 40: 1625-34.

(c) Farrell et al.; Licensee Bentham Open.

This is an open access article licensed under the terms of the Creative Commons Attribution Non-Commercial License (http://creativecommons.org/licenses/ by-nc/3.0/) which permits unrestricted, non-commercial use, distribution and reproduction in any medium, provided the work is properly cited. 